Published in final edited form as:

Breast Cancer Res Treat. 2018 January ; 167(1): 1-8. doi:10.1007/s10549-017-4490-3.

\title{
SOCIOECONOMIC STATUS AND BREAST CANCER TREATMENT
}

\author{
Marie S. Dreyer, BSBA, Ann B. Nattinger, MD, MPH, Emily L. McGinley, MS, MPH, and \\ Liliana E. Pezzin, PhD, JD \\ Department of Medicine and Center for Patient Care and Outcomes Research, Medical College \\ of Wisconsin, Milwaukee, WI
}

\begin{abstract}
Purpose-Evidence suggests substantial disparities in breast cancer survival by socioeconomic status (SES). We examine the extent to which receipt of newer, less invasive, or more effective treatments - a plausible source of disparities in survival - varies by SES among elderly women with early stage breast cancer.
\end{abstract}

Methods-Multivariate regression analyses applied to 11,368 women (age 66-90 years) identified from SEER-Medicare as having invasive breast cancer diagnosed in 2006-2009. Socioeconomic status was defined based on Medicaid enrollment and level of poverty of the census tract of residence. All analyses controlled for demographic, clinical health status, spatial and healthcare system characteristics.

Results-Poor and near-poor women were less likely than high SES women to receive sentinel lymph node biopsy and radiation after breast conserving surgery (BCS). Poor women were also less likely than near-poor or high SES women to receive any axillary surgery and adjuvant chemotherapy. There were no significant differences in use of aromatase inhibitors (AI) between poor and high SES women. However, near-poor women who initiated hormonal therapy were more likely to rely exclusively on tamoxifen, and less likely to use the more expensive but more effective AI when compared to both poor and high SES women.

Conclusions-Our results indicate that SES disparities in the receipt of treatments for incident breast cancer are both pervasive and substantial. These disparities remained despite women's geographic area of residence and extent of disease, suggesting important gaps in access to effective breast cancer care.

\section{Keywords}

breast cancer; adjuvant and neoadjuvant treatments; socioeconomic status; SEER-Medicare

\footnotetext{
Please address correspondence to: Liliana E. Pezzin, PhD JD, Medical College of Wisconsin, Center for Patient Care and Outcomes Research (PCOR), 8701 Watertown Plank Road, Suite H3100, Milwaukee, WI 53226, lpezzin@ mcw.edu, Phone: 414-456-8862, Fax: 414-955-6689.

Conflict of Interest: The authors declare that they have no conflict of interest.

Authors' Contributions: MSD and LEP are jointly responsible for the planning, conduct, and reporting of results; ELM conducted the statistical analyses; ABN provided clinical insights into variable construction and interpretation of results. As senior author, LEP is responsible for the overall content of the manuscript.

Ethical approval: This study has received ethical approval by the Medical College of Wisconsin/Froedtert Hospital Institutional Review Board \#5 as it satisfies requirements of 45 CFR 46.111.
} 


\section{INTRODUCTION}

Traditionally, population incidence and mortality rates of women with incident breast cancer were both lower among patients of lower socioeconomic status (SES). During the past three decades, however, this situation has changed: while the incidence of breast cancer remained lower among this group, mortality is now significantly higher among women of low SES relative to their high SES counterparts [1]. While the literature is replete of studies documenting disparities in breast cancer mortality by socioeconomic status [1-11], and notwithstanding the fact that eliminating such disparities is a national priority of HealthyPeople 2020, relatively little is known about its underlying source.

One plausible source of the observed disparities in breast cancer outcomes may be differential receipt of newer or more effective treatments by women of lower socioeconomic status. To the extent that, all else constant, there is variation in treatment attributed solely to a patient's socioeconomic status, the SES disparity in outcomes is likely to follow. Multiple studies have shown disparity in receipt of breast-conserving surgery (BCS) by SES [6,1216]. Relatively little is known, however, about disparities in other initial surgical (e.g. axillary surgery) or adjuvant therapies (e.g. chemotherapy, radiation, hormonal therapy). Few national studies have assessed breast cancer treatment disparities by socioeconomic status (SES), particularly within lower SES groups, in the United States.

The purpose of this study is to identify socioeconomic disparities in receipt of newer, less invasive, or more effective breast cancer treatments. We hypothesized that poor and nearpoor women with incident breast cancer are less likely than women of higher socioeconomic status to receive such recommended initial treatments and adjuvant therapies.

\section{Methods}

\section{Study Population and Data Sources}

The main data source for the study was the Surveillance, Epidemiology and End Results (SEER)-Medicare database, a linkage of two large population-based datasets that provide detailed information about elderly persons with cancer. Detailed clinical information, including extent of disease at presentation and cause of death, were available from the SEER program. Information on subject's cancer treatment as well as on demographic and socioeconomic characteristics were obtained from linked Medicare enrollment and claims files. These data were further supplemented by geocoded Census data [17].

The study sample consisted of elderly women between the ages of 66 and 90 years old identified from the SEER-Medicare database as having unilateral, invasive, stage I-III breast cancer diagnosed from July 2006 through December 2009. The sample was further restricted to those continuously enrolled in traditional (Fee-for-Service, FFS) Medicare for at least 12 months prior to their incident breast cancer diagnosis date in order to enable us to measure comorbidities. 


\section{Outcome Measures}

The main outcome of this study was receipt of specific breast cancer treatments.

Specifically, we evaluated whether an axillary surgery was performed and type of axillary surgery performed (sentinel lymph node dissection only (SLNB), axillary lymph node dissection only (ALND), both SLNB and ALND); receipt of radiation therapy, receipt of chemotherapy (neoadjuvant and adjuvant), and receipt of newer, more effective adjuvant endocrine therapy, based on the 2005 American Society of Clinical Oncology (ASCO) recommendations prevailing during the study period, which indicated that adjuvant treatment for postmenopausal women with hormone-receptor positive breast cancer should include an aromatase inhibitor [18]. Identification of 2005 ASCO guideline-discordant endocrine therapy regimen was based on patients' exclusive use of tamoxifen during the first four consecutive years following first endocrine therapy claim. Patients with at least one claim for any aromatase inhibitor (AI) during the 4-year period were considered guidelinecompliant and served as the reference group for the analysis.

\section{Socioeconomic Status}

Using individual-level enrollment information and geographic identifiers provided under a restricted Data Use Agreement with each of the 16 SEER registries, we constructed four mutually exclusive indicators of beneficiaries' socioeconomic status - poor, near poor, middle and high SES - as follows. First, we classified all Census tracts in states with a SEER registry into composite poverty quartiles based on per capita income (PCI), proportion of families below poverty line (POVT) and median household income (HHI) of tracts within each state. A study subject was then classified as "poor" if she was enrolled in her state of residence's Medicaid or a state buy-in program, regardless of Census tract of residence; "near-poor" if she was not enrolled in Medicaid or a state buy-in program but lived in a Census tract ranking in the highest quartile of poverty (defined as lowest quartile of both PCI and HHI and upper quartile of POVT); "high SES" if she was not enrolled in Medicaid or a state buy-in program and lived in a Census tract ranking in the lowest quartile of poverty (defined as upper quartile of both PCI and HHI and lowest quartile of POVT). Given its heterogeneity, we excluded from our analyses subjects belonging to the residual "middle income" group of women who were neither enrolled in a public subsidy program nor resided in a Census tract ranking in the lowest or highest quartile of poverty.

\section{Other covariates}

Women were classified according to their race/ethnicity as White non-Hispanic, Black/ African American non-Hispanic, Hispanic, Asian or other race/ethnicity. Marital status was married or not married. Age at diagnosis of first breast cancer and number of comorbidities, measured based on Medicare inpatient, outpatient, and provider claims data for the year preceding the incident breast cancer diagnosis based on the Klabunde algorithm [19] were both included as these factors have been shown to influence breast cancer treatment among older women [20]. Additional clinical variables included cancer stage, as classified by the American Joint Committee on Cancer (AJCC); hormone receptor status classified as combined status, estrogen receptor and/or progesterone receptor; and status of node examination as positive, negative or no exam. 
Other covariates included diagnosis year, SEER site (San Francisco, Connecticut, Detroit, Hawaii, Iowa, New Mexico, Seattle, Utah, Atlanta, San Jose, Los Angeles, Rural Georgia, Kentucky, Louisiana, New Jersey, Greater Georgia), and urban status of county of residence based on SEER classification, defined as large metropolitan area, metropolitan area, urban or rural.

\section{Statistical Analysis}

Baseline demographics are described with means and proportions in summary statistics.

Chi-squared trend tests were used to compare women of differing SES categories according to their demographic and clinical characteristics. Logistic regression models were used to determine the association between SES and receipt of any axillary surgery, SLNB or ALND, post-BCS radiation, neoadjuvant chemotherapy, adjuvant chemotherapy, and type of endocrine therapy (AI versus tamoxifen). As clinically indicated, analyses of adjuvant chemotherapy were restricted to women with stage II or stage III disease, analyses of postBCS radiation were restricted to women undergoing a breast conserving surgery, and analyses of hormone therapy were restricted to women with hormone positive disease.

Among those patients who did receive axillary surgery, multinomial logistic regression was used to evaluate the probability of receiving either SLNB only, SLNB and ALND, or ALND only. In addition to the covariates described above, which were included in all analyses, we controlled for node examination status in regressions where neoadjuvant chemotherapy and adjuvant chemotherapy were the outcomes of interest in order to control for direction of treatment based on extent of disease. Finally, hormone receptor status was included as an additional control variable when assessing factors associated with a woman's likelihood of receiving neoadjuvant chemotherapy, adjuvant chemotherapy and radiation. Odds ratios (ORs) and 95\% confidence intervals (CIs) were calculated. All analyses were performed using STATA 12.

\section{Results}

\section{Summary characteristics}

In total, 11,368 women with incident breast cancer were included in this study, of whom $34 \%$ were poor, $18 \%$ were near-poor and $48 \%$ were of high socioeconomic status. As shown in Table 1, women in our sample were predominantly white and older, reflecting both the racial distribution of Medicare beneficiaries and the increased incidence of breast cancer in white women. The majority of women lived in metropolitan areas and were unmarried. Nearly four out of every five women had hormone receptor positive disease and slightly more than half had stage I cancer. Majority of women had nodes examined, but the poor cohort were less likely to have had node examination as compared to near-poor and high SES women. Among women in the entire sample that did have node examination, majority were negative, which correlates to the predominance of stage I cancer. Poor women were more likely to have more than one comorbidity whereas high SES women were more likely to have no comorbidities. 


\section{Breast Cancer Treatments and Socioeconomic Status}

Table 2 shows the distribution of initial and adjuvant breast cancer treatments, overall and by socioeconomic status. The majority of women received some type of axillary surgery, with SLNB-ALND conversions being the most common axillary procedure performed. Women of high SES were more likely to receive strictly SLNB or to have an SLNB attempted while poor women were more likely to receive strictly ALND. Among women undergoing a BCS, a significant majority received radiation. Only $3 \%$ of the sample received neoadjuvant chemotherapy with no statistically significant difference in its receipt by SES. The majority did not receive adjuvant chemotherapy; among those who did, 36\% were near-poor, 34\% were of high SES and 30\% were poor.

One-quarter of women with hormone receptor positive disease who were enrolled in Medicare Part D did not receive any form of hormonal therapy. Among the subset of women with hormone receptor positive disease, who were enrolled in Medicare Part D and had begun hormonal therapy within 12 months from their surgery, the majority received an AI as part of their hormonal therapy regimen. This is consistent with the 2005 ASCO guidelines that prevailed during the study period.

Table 3 shows the relationship of socioeconomic status and receipt of specific breast cancer processes of care, adjusted for the covariates in Table 1. There were significant differences in the receipt of breast cancer treatments between poor women and women of high SES. Poor women were less likely than high SES women to receive the initial surgical treatments: axillary surgery, SLNB, and SLNB-ALND. Additionally, the poor were less likely to receive radiation after BCS and adjuvant chemotherapy. No significant differences were observed in receipt of neoadjuvant chemotherapy or the use of an AI between poor and high SES women.

Near-poor women were less likely than their high SES counterparts to receive an SLNB or to have an SLNB-attempted prior to the final ALND. Additionally, near-poor women with hormone positive disease were less likely to receive a hormonal therapy regimen that included an AI within the first four years of therapy when compared to hormone positive disease-women of high SES. There were no significant differences in the receipt of axillary surgery, radiation after BCS, neoadjuvant chemotherapy and adjuvant chemotherapy when compared to high SES women.

Socioeconomic status also influenced treatment receipt differences between lower SES levels, with poor women exhibiting statistically significant differences in treatment when compared to their near-poor counterparts (results not shown). All initial surgical treatment disparities, except for SLNB, remained even after controls for potential confounders. Poor women were less likely than near-poor women to receive axillary surgery (OR $0.69,95 \% \mathrm{CI}$ $0.58-0.82$ ) and SLNB-ALND (OR 0.84, 95\% CI 0.72-0.99); they were also less likely to receive radiation after BCS (OR 0.69, 95\% CI 0.54-0.87). Finally, poor women with stage II or III disease were less likely to receive adjuvant chemotherapy (OR 0.79, 95\% CI 0.64 0.97). In contrast, near-poor women were at greater disadvantage in terms of hormonal therapy as they were significantly more likely than poor women to rely exclusively on tamoxifen than AIs (OR 1.79, 95\% CI 1.20-2.66). 


\section{Discussion}

In this large, population-based sample of SEER-Medicare beneficiaries, there was a significant relationship between SES and receipt of initial surgical and adjuvant treatments for incident breast cancer, with poorer populations less likely to receive the same treatments as their high SES counterparts. Poor women were less likely than near-poor and high SES women to receive almost all treatments studied: axillary surgery, SLNB, post-BCS radiation and adjuvant chemotherapy. In addition, near-poor women were less likely than high SES women to either receive or have an SLNB attempted. There were no statistically significant differences in receipt of neoadjuvant therapy across the three SES groups, perhaps due to the low prevalence of neoadjuvant treatment overall during the study period (2006-2009).

An important, new finding concerns receipt of an $\mathrm{AI}$ as part of hormonal therapy regimen. For the majority of postmenopausal women with hormone receptor-positive disease, there are essentially two options for oral endocrine therapy. One is tamoxifen, a drug for which efficacy was initially demonstrated in the mid-1980's. The other choice is to use one of three aromatase inhibitor (AI) agents. Based on evidence suggesting that AIs, as initial therapy or after 2-3 years of tamoxifen, provided more effective control of breast cancer than tamoxifen alone among women with hormone receptor-positive disease, recommendations from the American Society of Clinical Oncology, published in 2005 and prevailing during the study period, suggested that adjuvant therapy for postmenopausal women with hormone receptor-positive breast cancer include an aromatase inhibitor [18,21-23]. Although the AI agents have recently become available as generic formulations with a considerably lower cost to the patient, for much of the past decade, these agents were significantly more costly than tamoxifen. Our results revealed that, for this particular breast cancer treatment, nearpoor women were the most disadvantaged group likely due to their ineligibility for Part Dspecific low-income subsidies despite relative poverty status.

The existing literature on SES disparities in breast cancer treatment often relies on small samples drawn from selected populations. A number of U.S. studies have concentrated on single state, county or metropolitan analyses to assess SES disparities in use of axillary surgery, type of breast surgery, chemotherapy and radiation [24-32]. Current larger population-based studies do provide a better idea of the national landscape; however, they tend to present piecemeal assessments rarely extending beyond the analysis of one or two processes of care, which alone only make up part of a treatment plan. Additionally, this study illustrates that disparities are not restricted to comparison between the extremes but persist between lower SES cohorts as well.

Several limitations of the study merit comment. Our sample is limited to elderly breast cancer beneficiaries enrolled in traditional (Fee-for-Service) Medicare as healthcare utilization data are not available for those enrolled in Medicare managed care. As of 2007, however, the vast majority (85\%) of Medicare beneficiaries were enrolled in such more traditional, non-capitated plans [33]. While the median onset of breast cancer in women is 62 years and women 65 years and older make up more than $40 \%$ of breast cancer patients [34], our results may not be generalizable to younger patients. Older women have been shown to have, on average, better survival than young women. The differences are at least 
partially attributable to more aggressive tumor grade and molecular subtypes in young women, and would imply that elderly women may not as often require as aggressive a treatment approach [35]. In addition, our findings of disparities by SES in receipt of hormonal therapy are further limited to the subset of women enrolled in the Medicare Part D prescription drug program. These findings may not apply to elderly breast cancer patients with a different source of coverage, such as private or employer-based pharmaceutical drug plans.

Our findings, based on a large sample of elderly Medicare beneficiaries, show substantial SES disparities in the receipt of certain breast cancer treatments. In regards to adjuvant therapy, our findings are consistent with the emerging literature indicating that women of low SES are less likely to receive post-BCS radiation $[6,16]$ and adjuvant chemotherapy $[6,36]$ than their wealthier counterparts. Our analyses also uncovered socioeconomic disparities in the receipt of other important breast cancer treatments, such as axillary surgery, SLNB and hormonal therapy. These results are of particular importance as these processes capture the continuum of breast cancer treatment. Axillary surgery, whether it is ALND or SLNB, is necessary for adequate biopsy. SLNB, specifically, is imperative to guide quality adjuvant treatment for the patient. Our study's finding of low SES women's lower likelihood of receiving SLNB is consistent with those recently reported by Chen et al and ReederHayes et al $[37,38]$.

Unlike the medical components (Parts A \& B) of Medicare, the U.S. federal health insurance program created in 1965 for people ages 65 and over regardless of income, coverage of prescription drugs through its Part D program relies on private plans within the various states. These plans are allowed wide discretion when setting plan features and prices. Our findings regarding near-poor women's exclusive reliance on tamoxifen for their hormonal therapy regimen raises concerns that, while Medicare Part D improved older patients' access to prescription drugs, it did relatively little to reduce socioeconomic variation in use of AIs [39-40], the (2005) ASCO-recommended breast cancer oral therapy, for women who were not eligible for the program's low-income subsidy despite relative poverty.

While racial disparities in breast cancer have dominated the literature on the subject and are certainly deserving of attention, this study's focus on socioeconomic disparities has the potential to play a vital role in helping eliminate inequalities among larger segments of the breast cancer population. Socioeconomic status is often intertwined with race or ethnicity and it can be challenging to parse out the effects of each on disparities in healthcare. Nonetheless, attention to alleviating socioeconomic disparities, which are more easily amenable to policy intervention, can also help reduce lingering disparities by race or ethnicity. For example, the highlighted finding of disparity in AI use among the near-poor population provides evidence that strategically subsidizing certain socioeconomic populations may promote optimal care.

In summary, our results indicate that SES disparities in the receipt of treatments for incident breast cancer are both pervasive and substantial. These disparities remained despite controls for women's geographic area of residence and extent of disease. This suggests important 
disparities-generating gaps in access to effective initial therapy and/or follow-up breast cancer care among women of lower socioeconomic status.

\section{Acknowledgments}

The collection of cancer incidence data used in this study was supported by the California Department of Public Health as part of the statewide cancer reporting program mandated by California Health and Safety Code Section 103885; the National Cancer Institute's Surveillance, Epidemiology and End Results Program under contract HHSN261201000140C awarded to the Cancer Prevention Institute of California, contract HHSN261201000035C awarded to the University of Southern California, and contract HHSN261201000034C awarded to the Public Health Institute; and the Centers for Disease Control and Prevention's National Program of Cancer Registries, under agreement \# U58DP003862-01 awarded to the California Department of Public Health. The ideas and opinions expressed herein are those of the author(s) and endorsement by the State of California Department of Public Health, the National Cancer Institute, and the Centers for Disease Control and Prevention or their Contractors and Subcontractors is not intended nor should be inferred. The authors acknowledge the efforts of the National Cancer Institute; the Office of Research, Development and Information, CMS; Information Management Services (IMS), Inc.; and the Surveillance, Epidemiology, and End Results (SEER) Program tumor registries in the creation of the SEER-Medicare database

The authors acknowledge the efforts of the National Cancer Institute; the Office of Research, Development and Information, CMS; Information Management Services (IMS), Inc.; and the Surveillance, Epidemiology, and End Results (SEER) Program tumor registries in the creation of the SEER-Medicare database.

Funding: The authors gratefully acknowledge funding by NCI under grant R01-CA 170945 and the American Cancer Society (RSG-13-070-01-CPHPS). No conflicts of interest disclosures from any authors.

\section{References}

1. Singh, Miller, Hankey, Edwards. Area socioeconomic variations in US cancer incidence, mortality, stage, treatment, and survival, 1975-1999. 2003.

2. Gordon NH, Crowe JP, Brumberg DJ, Berger NA. Socioeconomic factors and race in breast cancer recurrence and survival. Am J Epidemiol. 1992; 135(6):609-18. [PubMed: 1580237]

3. Gorey KM, Luginaah IN, Holowaty EJ, Fung KY, Hamm C. Breast cancer survival in ontario and california, 1998-2006: socioeconomic inequity remains much greater in the United States. Ann Epidemiol. 2009; 19(2):121-4. DOI: 10.1016/j.annepidem.2008.10.010 [PubMed: 19185806]

4. Sprague BL, Trentham-Dietz A, Gangnon RE, et al. Socioeconomic status and survival after an invasive breast cancer diagnosis. Cancer. 2011; 117(7):1542-51. DOI: 10.1002/cncr.25589 [PubMed: 21425155]

5. Harper S, Lynch J, Meersman SC, Breen N, Davis WW, Reichman MC. Trends in areasocioeconomic and race-ethnic disparities in breast cancer incidence, stage at diagnosis, screening, mortality, and survival among women ages 50 years and over (1987-2005). Cancer Epidemiol Biomarkers Prev. 2009; 18(1):121-31. DOI: 10.1158/1055-9965.EPI-08-0679 [PubMed: 19124489]

6. Byers T, Wolf H, Bauer K, et al. The impact of socioeconomic status on survival after cancer in the United States: findings from the National Program of Cancer Registries Patterns of Care Study. Cancer. 2008; 113(3):582-91. DOI: 10.1002/cncr.23567 [PubMed: 18613122]

7. Yu XQ. Socioeconomic disparities in breast cancer survival: relation to stage at diagnosis, treatment and race. BMC Cancer. 2009; 9:364.doi: 10.1186/1471-2407-9-364 [PubMed: 19828016]

8. Kish J, Yu M, Percy-Laurry A, Altekruse S. Racial and ethnic disparities in cancer survival by neighborhood socioeconomic status in Surveillance, Epidemiology, and End Results (SEER) Registries. Journal of the National Cancer Institute Monographs. 2014; 2014(49):236-43. DOI: 10.1093/jncimonographs/lgu020 [PubMed: 25417237]

9. Feinglass J, Rydzewski N, Yang A. The socioeconomic gradient in all-cause mortality for women with breast cancer: findings from the 1998 to 2006 National Cancer Data Base with follow-up through 2011. Annals of epidemiology. 2015; 25(8):549-55. DOI: 10.1016/j.annepidem. 2015.02.006 [PubMed: 25795226]

10. Bassett, Krieger. Social class and black-white differences in breast cancer survival. American journal of public health. 1986; 76(12):1400-3. [PubMed: 3777285] 
11. Singh, Williams, Siahpush. Socioeconomic, rural-urban, and racial inequalities in US cancer mortality: Part I-All cancers and lung cancer and Part II-Colorectal, prostate, breast, and cervical. 2012

12. Gilligan M, Kneusel R, Hoffmann R, Greer A, Nattinger A. Persistent differences in sociodemographic determinants of breast conserving treatment despite overall increased adoption. Medical care. 2002; 40(3):181-9. DOI: 10.1097/00005650-200203000-00002 [PubMed: 11880791]

13. Alderman A, Bynum J, Sutherland J, Birkmeyer N, Collins, Birkmeyer J. Surgical treatment of breast cancer among the elderly in the United States. Cancer. 2011; 117(4):698-704. DOI: 10.1002/cncr.25617 [PubMed: 20886632]

14. Smith GL, Xu Y, Shih Y-CTC, et al. Breast-conserving surgery in older patients with invasive breast cancer: current patterns of treatment across the United States. J Am Coll Surg. 2009; 209(4):425-433. e2. DOI: 10.1016/j.jamcollsurg.2009.06.363 [PubMed: 19801315]

15. Michalski T, Nattinger A. The influence of black race and socioeconomic status on the use of breast-conserving surgery for medicare beneficiaries. Cancer. 1997; 79(2):314-319. DOI: 10.1002/ (SICI)1097-0142(19970115)79:2<314::AID-CNCR14>3.0.CO;2-3 [PubMed: 9010104]

16. Mandelblatt J, Kerner J, Hadley J, et al. Variations in breast carcinoma treatment in older medicare beneficiaries. Cancer. 2002; 95(7):1401-1414. DOI: 10.1002/cncr.10825 [PubMed: 12237908]

17. U.S. Census Bureau. American Community Survey 2005-2009. 2014.

18. Winer EP, Hudis C, Burstein HJ, et al. American Society of Clinical Oncology technology assessment on the use of aromatase inhibitors as adjuvant therapy for postmenopausal women with hormone receptor-positive breast cancer: status report 2004. J Clin Oncol. 2005; 23(3):619-29. DOI: 10.1200/JCO.2005.09.121 [PubMed: 15545664]

19. Klabunde, Legler, Warren, Baldwin. A refined comorbidity measurement algorithm for claimsbased studies of breast, prostate, colorectal, and lung cancer patients. 2007.

20. Bouchardy C, Rapiti E, Fioretta G, et al. Undertreatment strongly decreases prognosis of breast cancer in elderly women. J Clin Oncol. 2003; 21(19):3580-7. DOI: 10.1200/JCO.2003.02.046 [PubMed: 12913099]

21. Howell A, Cuzick J, Baum M, et al. Results of the ATAC (Arimidex, Tamoxifen, Alone or in Combination) trial after completion of 5 years' adjuvant treatment for breast cancer. Lancet. 2005; 365(9453):60-2. DOI: 10.1016/S0140-6736(04)17666-6 [PubMed: 15639680]

22. Thürlimann B, Keshaviah A, Coates AS, et al. A comparison of letrozole and tamoxifen in postmenopausal women with early breast cancer. N Engl J Med. 2005; 353(26):2747-57. DOI: 10.1056/NEJMoa052258 [PubMed: 16382061]

23. Wang X, Du X. Socio-demographic and geographic variations in the utilization of hormone therapy in older women with breast cancer after Medicare Part-D coverage. Medical oncology (Northwood, London, England). 2015; 32(5):154.doi: 10.1007/s12032-015-0599-6

24. Dookeran K, Silva A, Warnecke R, Rauscher G. Race/ethnicity and disparities in mastectomy practice in the Breast Cancer Care in Chicago study. Annals of surgical oncology. 2015; 22(1):6674. DOI: 10.1245/s10434-014-3945-6 [PubMed: 25135847]

25. White A, Richardson L, Krontiras H, Pisu M. Socioeconomic Disparities in Breast Cancer Treatment Among Older Women. J Women's Heal. 2014; 23(4):335-341. DOI: 10.1089/jwh. 2013.4460

26. Nguyen B, Alawadi Z, Roife D, Kao L, Ko T, Wray C. Do Socioeconomic Factors and Race Determine the Likelihood of Breast-Conserving Surgery? Clin Breast Cancer. 2016; 16(4):e93e97. DOI: 10.1016/j.clbc.2016.05.008 [PubMed: 27297238]

27. Guy GP, Lipscomb J, Gillespie TW, Goodman M, Richardson LC, Ward KC. Variations in Guideline-Concordant Breast Cancer Adjuvant Therapy in Rural Georgia. Health Serv Res. 2015; 50(4):1088-108. DOI: 10.1111/1475-6773.12269 [PubMed: 25491350]

28. Gomez S, Press D, Lichtensztajn D, et al. Patient, hospital, and neighborhood factors associated with treatment of early-stage breast cancer among Asian American women in California. Cancer epidemiology, biomarkers \& prevention: a publication of the American Association for Cancer Research, cosponsored by the American Society of Preventive Oncology. 2012; 21(5):821-34. DOI: 10.1158/1055-9965.EPI-11-1143 
29. Olaya W, Wong J, Morgan J, Roy-Chowdhury S, Lum S. Disparities in the surgical management of women with stage I breast cancer. The American surgeon. 2009; 75(10):869-72. [PubMed: 19886124]

30. Olaya W, Wong J, Morgan J, et al. Factors Associated with Variance in Compliance with a Sentinel Lymph Node Dissection Quality Measure in Early-Stage Breast Cancer. Annals of Surgical Oncology. 2010; 17(3):297-302. DOI: 10.1245/s10434-010-1248-0 [PubMed: 20853050]

31. Bradley C, Given C, Roberts C. Race, Socioeconomic Status, and Breast Cancer Treatment and Survival. J Natl Cancer I. 2002; 94(7):490-496. DOI: 10.1093/jnci/94.7.490

32. McGinnis, Menck, Eyre, Bland. National Cancer Data Base survey of breast cancer management for patients from low income zip codes. 2000; doi: 10.1002/ (SICI)1097-0142(20000215)88:4<933::AID-CNCR25>3.0.CO;2-I

33. Montgomery, Pope, Greenwald, Kautter. Medicare Advantage Plan Availability, Premiums and Benefits, and Beneficiary Enrollment in 2007. 2010.

34. Turner N, Zafarana E, Becheri D, Mottino G, Biganzoli L. Breast cancer in the elderly: which lessons have we learned? Future Oncol. 2013; 9(12):1871-81. DOI: 10.2217/fon.13.140 [PubMed: 24295417]

35. Partridge, Ann H., et al. Subtype-Dependent Relationship Between Young Age At Diagnosis And Breast Cancer Survival. Journal of Clinical Oncology. 2016; 34(27):3308-3314. [PubMed: 27480155]

36. Bhargava A, Du X. Racial and socioeconomic disparities in adjuvant chemotherapy for older women with lymph node-positive, operable breast cancer. Cancer. 2009; 115(13):2999-3008. DOI: 10.1002/cncr.24363 [PubMed: 19452539]

37. Chen A, Halpern M, Schrag N, Stewart A, Leitch M, Ward E. Disparities and trends in sentinel lymph node biopsy among early-stage breast cancer patients (1998-2005). Journal of the National Cancer Institute. 2008; 100(7):462-74. DOI: 10.1093/jnci/djn057 [PubMed: 18364506]

38. Reeder-Hayes K, Bainbridge J, Meyer A. Race and age disparities in receipt of sentinel lymph node biopsy for early-stage breast cancer. 2011; doi: 10.1007/s10549-011-1398-1

39. Neuner, Joan M., et al. The introduction of generic aromatase inhibitors and treatment adherence among Medicare D enrollees. Journal of the National Cancer Institute. 2015; 107(8):djv130.39. [PubMed: 25971298]

40. Riley, Gerald F., et al. Endocrine therapy use among elderly hormone receptor-positive breast cancer patients enrolled in Medicare Part D. Medicare \& medicaid research review. 2011; 1:4. 


\section{Table 1}

Sample Statistics, Overall and by Socioeconomic Status ${ }^{a}$

\begin{tabular}{|c|c|c|c|c|}
\hline & Total & Poor & Near poor & High SES \\
\hline Total number & 11,368 & 3,869 & 2,014 & 5,485 \\
\hline Age, mean (SD) & $76(6)$ & $76(6)$ & $76(6)$ & $75(6)$ \\
\hline \multicolumn{5}{|l|}{ Race, $\%$} \\
\hline White & 80 & 63 & 71 & 95 \\
\hline Black & 12 & 19 & 24 & 2 \\
\hline Asian & 4 & 9 & 1 & 1 \\
\hline Hispanic & 2 & 6 & 1 & 1 \\
\hline Native & 1 & 1 & 1 & 0 \\
\hline Other & 2 & 2 & 2 & 2 \\
\hline \multicolumn{5}{|c|}{ Marital Status, \% } \\
\hline Unmarried & 61 & 80 & 61 & 48 \\
\hline Married & 39 & 20 & 39 & 52 \\
\hline \multicolumn{5}{|l|}{ Comorbidity, \% } \\
\hline None & 48 & 31 & 48 & 61 \\
\hline 1 & 27 & 29 & 28 & 25 \\
\hline $2+$ & 25 & 40 & 24 & 14 \\
\hline \multicolumn{5}{|l|}{ SEER Stage, \% } \\
\hline I & 52 & 42 & 50 & 59 \\
\hline II & 32 & 37 & 33 & 29 \\
\hline III & 11 & 14 & 11 & 8 \\
\hline \multicolumn{5}{|c|}{ Hormone Receptor Status, \% } \\
\hline Unknown & 6 & 9 & 7 & 4 \\
\hline Negative & 14 & 16 & 17 & 13 \\
\hline Positive & 79 & 76 & 76 & 83 \\
\hline \multicolumn{5}{|l|}{ Node status, $\%$} \\
\hline Negative & 60 & 52 & 60 & 66 \\
\hline Positive & 25 & 29 & 26 & 21 \\
\hline No exam & 14 & 18 & 13 & 12 \\
\hline \multicolumn{5}{|l|}{ Urban area, \% } \\
\hline Large metro & 60 & 55 & 37 & 72 \\
\hline Metro & 28 & 27 & 35 & 26 \\
\hline Urban & 11 & 15 & 25 & 2 \\
\hline Rural & 1 & 3 & 3 & 1 \\
\hline
\end{tabular}

${ }^{a}$ All differences were statistically significant at $\mathrm{p}<0.05$ level. 
Table 2

Unadjusted Rate of Breast Cancer Treatments, Overall and by Socioeconomic Status

\begin{tabular}{|c|c|c|c|c|}
\hline & Total & Poor & Near poor & High SES \\
\hline Total number & 11,368 & 3,869 & 2,014 & 5,485 \\
\hline \multicolumn{5}{|l|}{ Axillary Surgery, \% } \\
\hline No Axillary Surgery & 13 & 17 & 12 & 11 \\
\hline Sentinel Lymph Node Biopsy only (SLNB) & 33 & 26 & 31 & 38 \\
\hline Axillary Lymph Node Dissection only (ALND) & 17 & 26 & 22 & 9 \\
\hline SLNB-ALND conversion & 37 & 31 & 35 & 42 \\
\hline Post breast-conserving surgery radiation $1, \%$ & 81 & 75 & 79 & 85 \\
\hline Neoadjuvant Chemotherapy, $\%$ & 3 & 3 & 3 & 3 \\
\hline Adjuvant Chemotherapy ${ }^{2}, \%$ & 33 & 30 & 36 & 34 \\
\hline \multicolumn{5}{|l|}{ Hormonal Therapy, \% } \\
\hline Exclusively Tamoxifen ${ }^{3}$ & 12 & 11 & 20 & 10 \\
\hline Included an Aromatase Inhibitor ${ }^{3}$ & 88 & 89 & 80 & 90 \\
\hline
\end{tabular}

${ }^{1}$ Among the subset of women who underwent a breast-conserving surgery.

2 Among the subset of women with stage II or stage III disease.

3 Among the subset of women with hormone receptor positive disease, who were enrolled in Medicare Part D during the study follow-up period and who had begun hormonal therapy within 12 months from their surgery. 
Table 3

Odds of Receiving Treatment, Relative to High SES Breast Cancer Patients

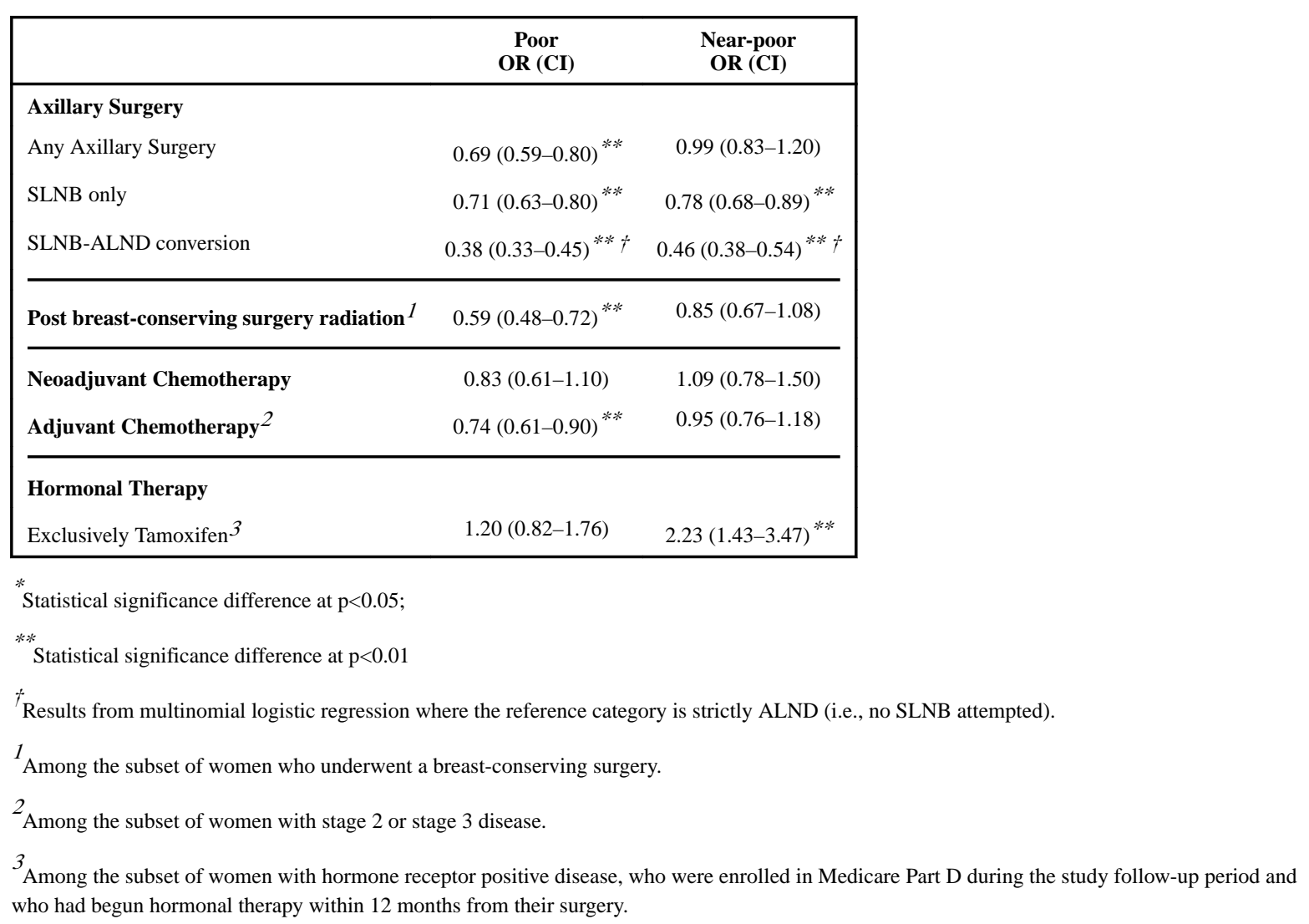

